International Mathematical Forum, 2, 2007, no. 23, 1129 - 1140

\title{
Characterizations of the Isometries and Construction of the Orbits in the Hyperbolic Plane
}

\author{
Domingo Gámez \\ Dpt. of Applied Math. \\ University of Granada \\ E-18071 Granada, Spain. \\ domingo@ugr.es \\ Miguel Pasadas \\ Dpt. of Applied Math. \\ University of Granada \\ E-18071 Granada, Spain. \\ mpasadas@ugr.es \\ Ceferino Ruiz \\ Dpt. of Geometry and Topology \\ University of Granada \\ E-18071 Granada, Spain. \\ ruiz@ugr.es
}

\begin{abstract}
In this work we define the isometries of the hyperbolic plane from its fixed points, and we characterize the orbits determined by some of them as rotations with a fixed center, limit rotations with a fixed point in the infinity line and translations along a line. These orbits are called, respectively, circumferences, horocycles and hypercycles. We illustrate an exhaustive classification of the isometries by means of the study of their fixed points. Some methods for building the aforementioned orbits are determined and some algorithms for their implementation are described. We used these algorithms to create a package of modules for Mathematica software, called Hyperbol, for the representation of such orbits, and we solve some constructive problems related with them.
\end{abstract}

Mathematics Subject Classification: 20H15, 51M15, 68W01, 20H10, 51M10, 68A10. 
Keywords: Hyperbolic geometry, isometry, orbit, algorithms.

\section{Notations and Preliminaries}

Let $\mathbb{C}^{+}$be the open upper half-plane $\mathbb{C}^{+}=\{z \in \mathbb{C} \mid \operatorname{Im} z>0\}$ endowed with the metric $d s=\frac{|d z|}{I m z}[7]$. We denote by $H^{2}$ this set with such metric. The lines in $H^{2}$ are euclidean half-circumferences centered at a point in the boundary of $H^{2}$ which corresponds to the parametrization: $x(t)=r \cos t+k_{1}, y(t)=$ $r \sin t, t \in(0, \pi)$; and euclidean half-lines orthogonal to that boundary with the parametrization: $x(t)=k_{2}, y(t)=t,(t>0)$. We have, making use of the Moebius transformation [9], that the group of isometries of $H^{2}$ preserving the orientation is given by

$$
I s o^{+}\left(H^{2}\right)=\left\{g: H^{2} \longrightarrow H^{2} \mid g(z)=\frac{a z+b}{c z+d} ; a, b, c, d \in \mathbb{R} ; a d-b c=1\right\} .
$$

The isometries of $H^{2}$ not preserving the orientation are the composition of the isometries preserving the orientation plus a fixed isometry that does not preserve the orientation.

Usually, the reflection on the imaginary axis $h(z)=-\bar{z}$ is used as an isometry not preserving the orientation, in order to obtain the isometries not preserving the orientation from the isometries preserving the orientation.

We denote by $\mathrm{Iso}^{-}\left(H^{2}\right)$ the set of the isometries of $H^{2}$ not preserving the orientation given by

$$
\left\{g^{\prime}: H^{2} \longrightarrow H^{2} \mid g^{\prime}(z)=\frac{a^{\prime} \bar{z}+b^{\prime}}{c^{\prime} \bar{z}+d^{\prime}} ; a^{\prime}, b^{\prime}, c^{\prime}, d^{\prime} \in \mathbb{R} ; a^{\prime} d^{\prime}-b^{\prime} c^{\prime}=-1\right\} \text {. }
$$

Let $D=\{z \in \mathbb{C} ;|z|<1\}$, i.e., the image of $\mathbb{C}^{+}$by the Cayley transformation $f_{c}: \mathbb{C}^{+} \longrightarrow D$, defined by $f_{c}(z)=\frac{z-i}{z+i}$. By means of $f_{c}$, the metric on $H^{2}$ is transformed into $d s=2 \frac{|d z|}{1-|z|^{2}}$ for $D$, and with that metric, it will be denoted by $D^{2}$. We can check that [3]

$$
I s o^{+}\left(D^{2}\right)=\left\{s: D^{2} \longrightarrow D^{2} \mid s(z)=\frac{\alpha z+\bar{\beta}}{\beta z+\bar{\alpha}} ; \alpha, \beta \in \mathbb{C}, \alpha \bar{\alpha}-\beta \bar{\beta}=1\right\} .
$$

The set $I s o^{-}\left(D^{2}\right)$ is given by

$$
I s o^{-}\left(D^{2}\right)=\left\{t: D^{2} \longrightarrow D^{2} \mid t(z)=\frac{\gamma \bar{z}+\bar{\delta}}{\delta \bar{z}+\bar{\gamma}} ; \gamma, \delta \in \mathbb{C}, \gamma \bar{\gamma}-\delta \bar{\delta}=1\right\} .
$$




\section{Rotations, limit rotations, translations, re- flections and glide reflections}

We define as follows the distinct types of isometries in $H^{2}$ and $D^{2}$ preserving and inverting the orientation.

Definition 2.1 Consider a transformation $g: H^{2} \rightarrow H^{2}\left(s: D^{2} \rightarrow D^{2}\right)$.

a) This transformation is a rotation centered at $z_{0} \in H^{2}\left(z_{0} \in D^{2}\right)$ if it is an isometry preserving the orientation and it only fixes the point $z_{0}$. In this case, $z_{0}$ is called the rotation center.

b) This transformation is a limit rotation centered at $z_{0}$ belonging to the infinity line, if it is an isometry preserving the orientation and it only fixes the point $z_{0}$. In this case, $z_{0}$ is called limit rotation center.

c) This transformation is a translation either on or with respect to a line $l$ if it is an isometry that preserves the orientation and fixes two points in the infinity line, which are those obtained as the intersection of the infinity line with $l$, called the translation line.

Definition 2.2 Consider a transformation $g^{\prime}: H^{2} \rightarrow H^{2}\left(t: D^{2} \rightarrow D^{2}\right)$

a) This transformation is a reflection of axis the line $l$ if it is an isometry that inverts the orientation, fixes the points of $l$ and interchanges the two connected components of its complement.

b) This transformation is a glide reflection of reflection line $l$ if it is an isometry that inverts the orientation, that does not fix the points of $H^{2}$ $\left(D^{2}\right)$ and that fixes two points of the infinity line, which are those obtained as the intersection of this line with $l$.

By using the fixed points of the isometries we can carry out an exhaustive and systematical classification of them in $H^{2}$ and $D^{2}$ from the following results $[2]$.

Theorem 2.3 Let $g \in I s o^{+}\left(H^{2}\right)$ and $s \in I s o^{+}\left(D^{2}\right)$.

i) $g$ is a rotation on $H^{2}$ if and only if $|a+d|<2$.

ii) $g$ is a limit rotation or the identity on $H^{2}$ if and only if $|a+d|=2$.

iii) $g$ is a translation on $H^{2}$ if and only if $|a+d|>2$.

iv) $s$ is a rotation on $D^{2}$ if and only if $\mid$ Reo $\mid<1$. 
v) $s$ is a limit rotation or the identity on $D^{2}$ if and only if $|R e \alpha|=1$.

vi) $s$ is a translation on $D^{2}$ if and only if $\mid$ Reo $\mid>1$.

Theorem 2.4 Let $g^{\prime} \in I s o^{-}\left(H^{2}\right)$ and $t \in I s o^{-}\left(D^{2}\right)$.

i) $g^{\prime}$ is a reflection on $H^{2}$ if and only if $\left|a^{\prime}+d^{\prime}\right|=0$.

ii) $g^{\prime}$ is a glide reflection on $H^{2}$ if and only if $\left|a^{\prime}+d^{\prime}\right| \neq 0$.

iii) $t$ is a reflection on $D^{2}$ if and only if $\operatorname{Re} \delta=0$.

iv) $t$ is a glide reflection on $D^{2}$ if and only if $R e \delta \neq 0$.

\section{Construction of the circumference}

Definition 3.1 A circumference is the set of images of a point $Q \in H^{2}$ $\left(D^{2}\right)$ by means of the reflection according to all the lines through a given point $A \in H^{2}\left(D^{2}\right)$, which is called the center of the circumference.

In the circumference case, we can deduce the following properties:

a) The circumferences centered at $A(a, b)$ are the orbits of the group of all elliptic isometries with a fixed point $A$ (rotations with center at $A$ ).

b) The circumference is a euclidean circumference contained in $\mathbb{C}^{+}$(respectively $D)$.

c) A circumference is a curve orthogonal to each line that contains $A$.

The construction of the circumference with center $A(a, b)$ and radius $R>0$ can be realized in $H^{2}$ and $D^{2}$, respectively, as follows.

Draw the line $x=a$, calculate the point $C$ on this line whose second coordinate is greater than the second coordinate of $A$ and whose distance to $A$ is $R$, obtaining $C\left(a, b e^{R}\right)[6]$. Let us consider any line through $A$, and on it we determine a point $B(c, d)$ whose distance to $A$ is $R$. Finally, we draw the euclidian mediatrix of the euclidian segment line $\overline{B C}$, whose equation is

$$
y-\frac{b e^{R}+d}{2}=\frac{a-c}{d-b e^{R}}\left(x-\frac{a+c}{2}\right) .
$$

The intersection of that mediatrix with $x=a$ gives us the center $E$ of the euclidian circumference whose coordinates are

$$
\left(a, \frac{d^{2}-b^{2} e^{2 R}+(a-c)^{2}}{2\left(d-b e^{R}\right)}\right) .
$$




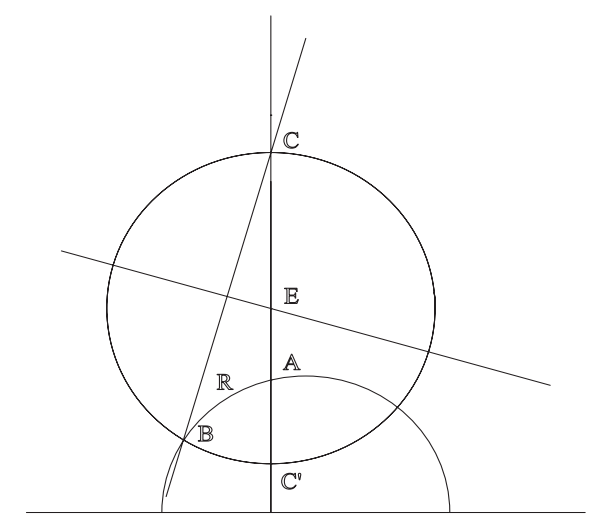

Figure 1: Construction of the circumference in $H^{2}$

Then, the circumference of center $A$ and radius $R$ is the euclidian circumference of center $E$ and radius $\overline{E C}$. The intersection of the line $x=a$ and the circumference is the points $C$ and $C^{\prime}=\left(a, b e^{-R}\right)$ [4]. The euclidean radius of this circumference is $b \sinh (R)$. Hence, the euclidean center $E$ can be also expressed $(a, b \cosh (R))$, and from (1) we deduce

$$
R=\operatorname{arccosh}\left(\frac{b^{2}+a^{2}+(a-c)^{2}}{2 b d}\right)=\mathrm{d}(A, B),
$$

Thus, we have obtained the known expression of the distance between two points from the circumference construction.

We can construct the orbits of the distinct isometries in $D^{2}$ in the following way:

a) Transform the points of $D^{2}$ to points of $H^{2}$ by $f_{c}^{-1}$.

b) Construct the corresponding orbit in $H^{2}$.

c) Transform the orbit of $H^{2}$ to the corresponding orbit in $D^{2}$.

Given the radius $R$ and the center $A$ in $D^{2}$, let $\widetilde{A}=f_{c}^{-1}(A)$. We determine three equidistant points $R$ from $\widetilde{A}$, from their images by $f_{c}$, in $D^{2}$, calculate the center $E$ and the radius of the euclidean circumference contained in $D$.

Namely, given $R>0$ and the point $A \in D^{2}$, let $\widetilde{A}=f_{c}^{-1}(A)$. We determine three points $\widetilde{B}, \widetilde{C}$ and $\widetilde{F}$ of the circumference of $H^{2}$ with center $\widetilde{A}$ and radius $R$. Then, we consider the points $\left.f_{c}(\widetilde{B})=B, f_{c} \widetilde{C}\right)=C$ and $f_{c}(\widetilde{F})=F$ of $D^{2}$. The circumference of center $A$ and radius $R$ in $D^{2}$ is the euclidian circumference through the points $B, C$ and $F$. 


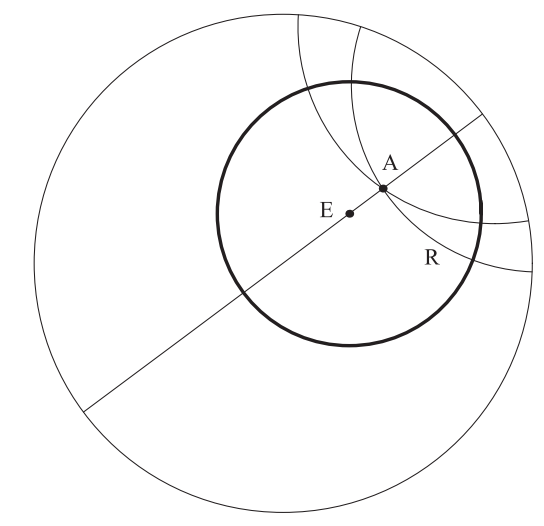

Figure 2: Construction of the circumference in $D^{2}$.

\section{Construction of the horocycle}

Definition 4.1 The horocycle with center at point $P$ of the infinity line is the set of all images of a fixed point $Q$ by the reflections with respect to the asymptotic lines of the point $P$.

We can obtain the following properties for the horocycles:

a) The horocycles can be characterized as the orbits for the group of parabolic isometries (limit rotations) with point $P$ belonging to the infinity line.

b) The horocycle is obtained from the circumference as a limit case. Namely, in $H^{2}$, if we move the center of the circumference through a given line to a point $P(p, 0)$ of the infinity line, the horocycle is a euclidean circumference contained in $\mathbb{C}^{+}$and tangent to the abscises axis at that point, and if we move the center to the infinity point of the infinity line, the horocycle is a euclidean line contained in $\mathbb{C}^{+}$whose equation is $y=k$. On the other hand, in $D^{2}$, if we move the center of the circumference through a given line to a point in $\operatorname{fr}\left(D^{2}\right)$, the horocycle is a euclidean circumference contained in the unit disk $D$ tangent to the boundary of $D$, at that point.

c) It is easy to check that the horocycle orthogonally intersects all the lines of the asymptotic pencil at $P$, being equal the distance between the two of these with center at $P$.

The construction of the horocycle through the points $A(a, b)$ in the hyperbolic plane and $P$ in the infinity line can be achieved in $H^{2}$ and $D^{2}$, respectively, as follows.

In $H^{2}$ we distinguished the following cases: 
1.- If $P(p, 0)$ is a point of the infinity line, then it is obvious that the center of the euclidean circumference is $C(p, q)$; where $q=\frac{(a-p)^{2}+b^{2}}{2 b}$, which coincides with its radius.

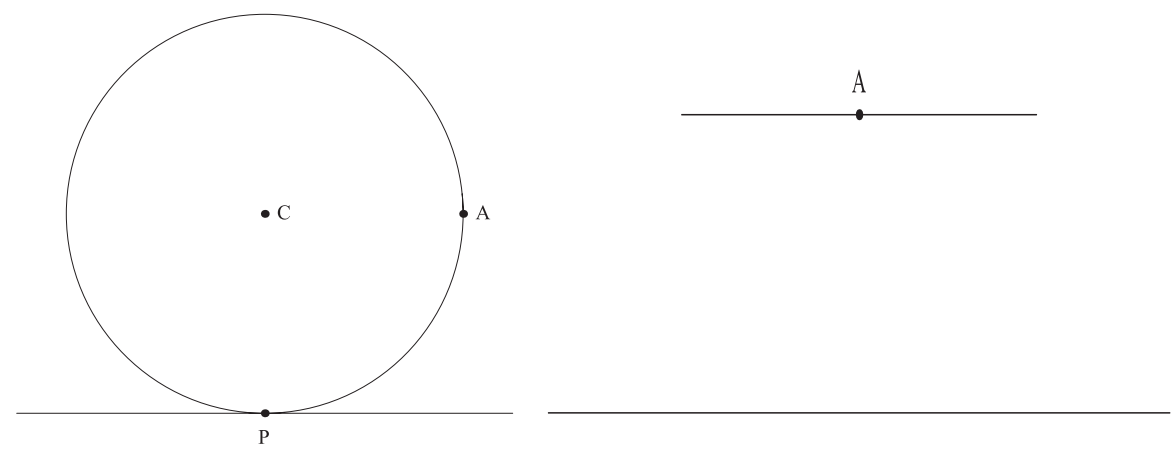

Figure 3: Horocycle in $H^{2}$ with center in the infinity line and in the infinity, respectively, from left to right.

2.- If $P$ is infinity, the horocycle through $A$ with center $P$ is the euclidean line contained in $\mathbb{C}^{+}$with equation $y=b$.

In $D^{2}$, let $P(p, q) \in f r\left(D^{2}\right)$ and $A \in D^{2}$. The horocycle with center $P$ through $A$ is the euclidean circumference through $A$ and $P$ such that is tangent to the boundary of $D^{2}$ at $P$.
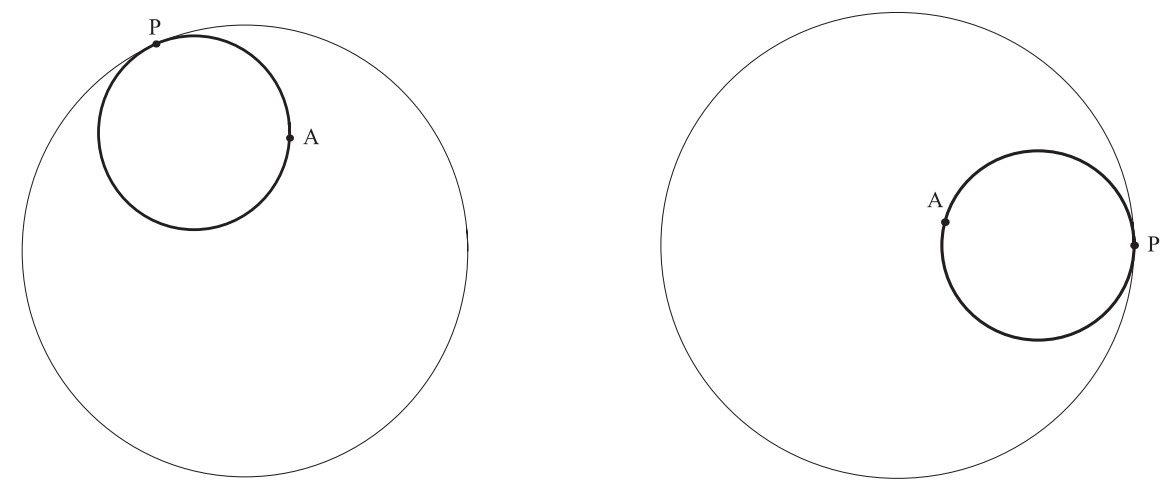

Figure 4: Horocycle with center in a point of the infinity line and in the infinity, respectively from left to right.

In the same context, we consider the following problem: Calculate the horocycles through two given points $A(a, b)$ and $B(c, d)$.

We now distinguish the cases $H^{2}$ and $D^{2}$, respectively, as follows: 
1.- If $b \neq d$, taking into account that the equation of the euclidean line which is orthogonal to the euclidean segment $\overline{A B}$ and which contains its middle point is

$$
(c-a) x+(d-b) y-\frac{d^{2}-b^{2}}{2}-\frac{c^{2}-a^{2}}{2}=0,
$$

and that the point $A$ belongs to the euclidean circumferences, then the solution of the system of indeterminates $p$ and $q$

$$
\left\{\begin{array}{l}
(c-a) p+(d-b) q-\frac{d^{2}-b^{2}}{2}-\frac{c^{2}-a^{2}}{2}=0 \\
p^{2}-2 a p+a^{2}-2 b q+b^{2}=0
\end{array}\right.
$$

determines the centers $C$ and $C^{\prime}$ of such euclidean circumferences.
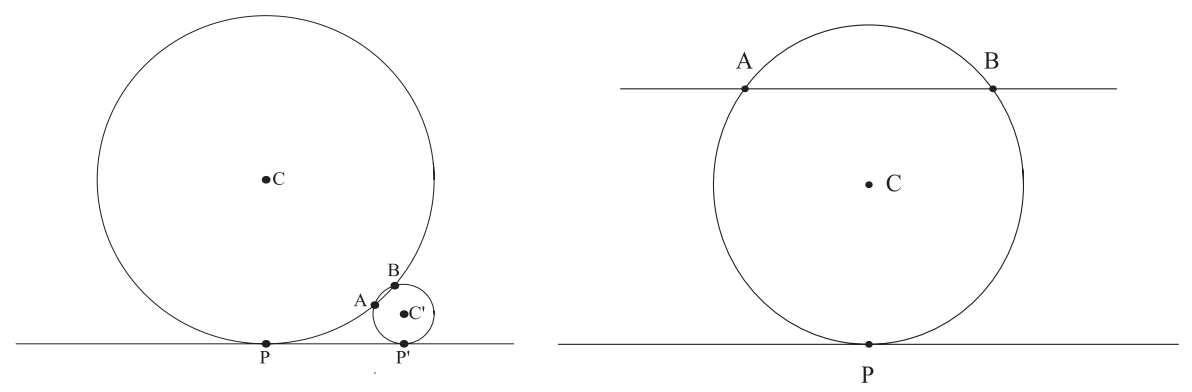

Figure 5: Horocycles through two points $A(a, b)$ and $B(c . d)$ with $b \neq d$ and $b=d$, respectively, from left to right.

2.- If $b=d$, we obtain the horocycle, which is a euclidean circumference whose center and radius are determined by the system (2) taking $b=d$, and the horocycle given by the euclidean line $y=b$.

In $D^{2}$, the horocycles through the points $A$ and $B$ are the tangent circumferences to the boundary of $D^{2}$ through these points.

\section{Construction of the hypercycle}

Definition 5.1 We define the hypercycle determined by a point $Q$ and a line $l$ in the hyperbolic plane as the set of images of $Q$ by means of reflections with respect to all the orthogonal lines to $l$. The line $l$ is called translation line.

The following properties of the hypercycles are important:

a) The hypercycles are the orbits of the group of all hyperbolic isometries fixing the two points of the intersection of the line $l$ and the infinity line. 

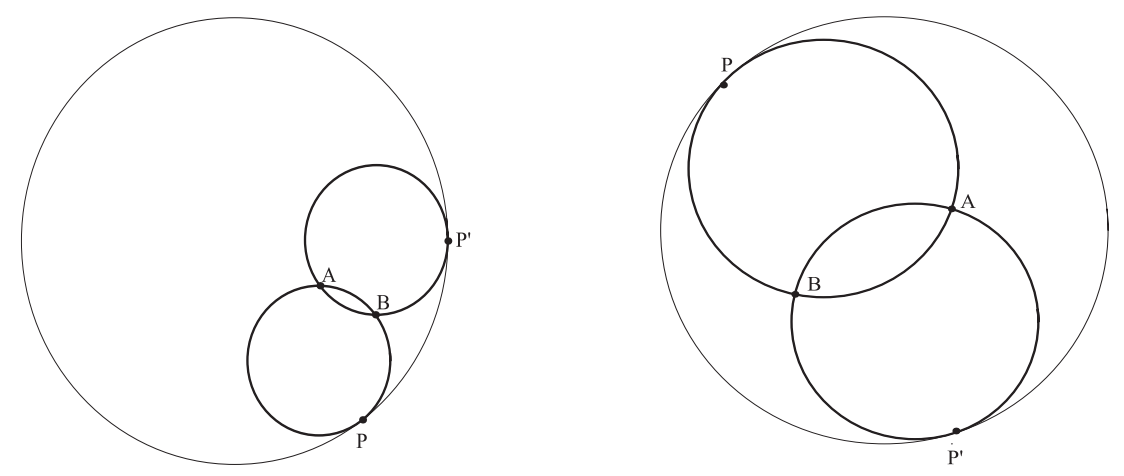

Figure 6: Horocycles through two points in $D^{2}$.

b) In $H^{2}$ : If the translation line meets the infinity line at two points $A(a, 0)$ and $B(b, 0)$, the hypercycle is an arc of a euclidean circumference through these points. If the translation line meets the infinity line at a point $A(a, 0)$ as well as the infinity point, the hypercycle is a euclidean halfline whose origin point is $A$.

c) In $D^{2}$ : A hypercycle is an arc of a euclidean circumference through two points of the infinity line determined by the translation line.

d) If the point $Q$ belongs to the translation line, the hypercycle coincides with that line.

e) The distance between any point of the translation line and the hypercycle, measured on the orthogonal line, is the same, that is, is constant.

f) Any two hypercycles of the same pencil are "parallels" in the sense that the distance of one of them to the other is constant.

The construction of the unique hyperciycle through two points $A$ and $B$ of the infinity line and a third point $C$ of the hyperbolic plane can be achieved in $H^{2}$ and $D^{2}$, respectively, as follows.

In $H^{2}$ we distinguished the following cases:

1.- If $A$ and $B$ are points of the infinity line with coordinates $(a, 0)$ and $(b, 0)$, respectively, with $a<b$ and $C(c, d) \in H^{2}$, the hypercycle through $A, B$ and $C$ is the intersection with $\mathbb{C}^{+}$of the euclidean circumference whose center $E$ and radius $r$ are obtained solving the system

$$
\left\{\begin{array}{l}
(a-p)^{2}+q^{2}=r^{2} \\
(b-p)^{2}+q^{2}=r^{2} \\
(c-p)^{2}+(d-q)^{2}=r^{2}
\end{array}\right.
$$



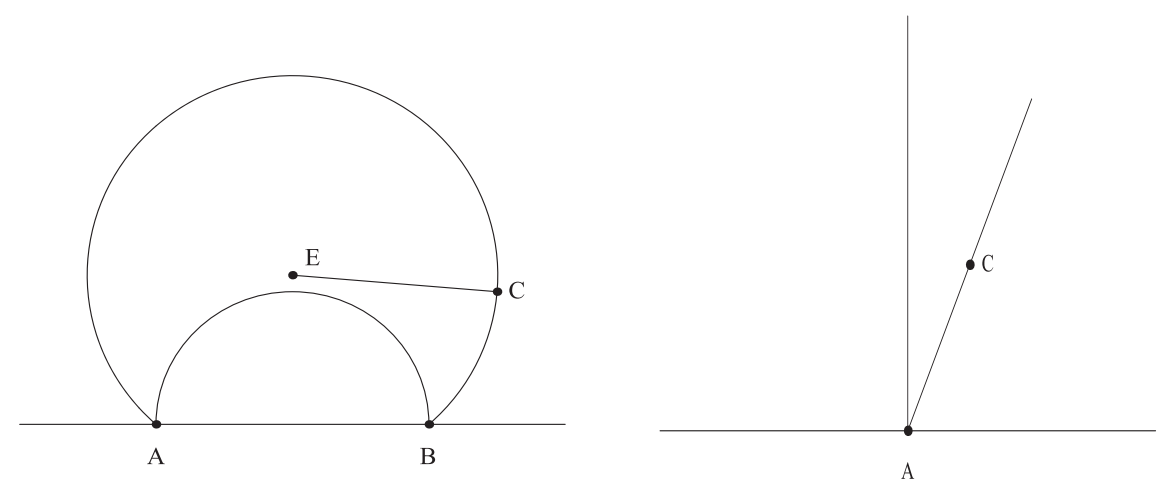

Figure 7: Hypercycle in $H^{2}$ through points $A, B$ and $C$, and through points $A, C$ and infinity.

The center $E$ has coordinates: $\left(\frac{a+b}{2}, \frac{(a-c)(b-c)+d^{2}}{2 d}\right)$,

and its radius is: $\frac{\sqrt{\left((a-c)^{2}+d^{2}\right)\left((b-c)^{2}+d^{2}\right)}}{2 d}$.

2.- If $A(a, 0), B$ is the infinity, and $C(c, d) \in H^{2}$, the hypercycle is the euclidean half-line of equation: $y=\frac{d}{c-a}(x-a)$, if $c \neq a$, or $x=a$, if $c=a$.

Now, let $A, B \in \operatorname{fr}\left(D^{2}\right)$ and $C \in D^{2}$. Then the hyperciycle through $A, B$ and $C$ is the arc of the euclidean circumference through the three cited points.
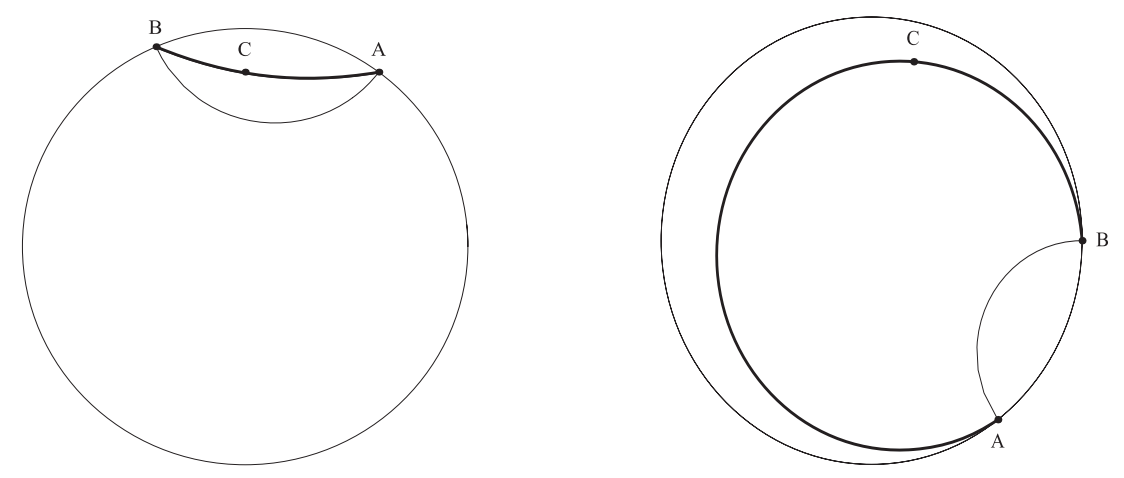

Figure 8: Hypercycles in $D^{2}$ through points $A, B$ and $C$. 


\section{Conclusions}

The main contributions of this work to the study of the orbits of some isometries in the hyperbolic plane are the following:

1.- The systematic characterization of the isometries as rotations, limit rotations, translations, reflections or glide reflections through the study of their fixed points. Also, the determination of the algorithms for the application of these isometries.

2.- The determination of precise algorithms for the construction of the orbits, circumferences, horocycles and hypercycles from different initial data.

3.- The implementation of these algorithms in a set of programming modules with the software Mathematica, included in a package named Hyperbol (software available at http://www.ugr.es/local/ruiz/software.htm) that we have designed for the numeric and graphic resolution of a wide array of constructive problems in the hyperbolic plane, in $H^{2}$ and $D^{2}$.

ACKNOWLEDGEMENTS. Research partially supported by Grants of PAI: FQM-191 and FQM-324

\section{References}

[1] Benedetti, R. and Petronio, C., 1992. Lectures on hyperbolic geometry. Springer- Verlag. Berlin.

[2] Gámez, D., 2001. Construcciones en Geometría Hiperbólica y teselaciones mediante grupos NEC poligonales. Algoritmos de automatización. PhD Thesis, University of Granada.

[3] Gámez, D., Pasadas, M., Pérez, R., Ruiz, C., 2001. Hyperbolic plane tessellations. Proccedings of the VI Journèes Zaragoza-Pau de Mathematiques Appliquèes et Statistique. Publ. de l'Univ. de Pau, Pau, 257-264.

[4] Gámez, D., Pasadas, M., Pérez, R., Ruiz, C., 2001. Regla y compás hiperbólicos electrónicos para teselar. Proceedings of the I Meeting of Andalusian Mathematicians: I E.M.A.. Vo. 2, Univ. Sevilla, Secr. Publ., Sevilla, 467-474.

[5] Gámez, D., Pasadas, M., Pérez, R., Ruiz, C., 2003. Orbits in the hyperbolic plane. Monografías del Seminario Matemático García de Galdeano. VII Jornadas Zaragoza-Pau de Mathemática Aplicada y Estadistica. Publ. Univ. de Zaragoza, Zaragoza, 297-305. 
[6] Gámez, D., Pasadas, M., Pérez, R., Ruiz, C., 2002. The Lambert quadrilateral and tesselation in the hyperbolic plane. Int. Math. J. 2 (8), 777795 .

[7] Iversen, B., 1992. Hyperbolic geometry. Cambridge University Press, Cambridge.

[8] Lenz, H., 1967. Nichteuklidische Geometrie, BI. Mannhein.

[9] Magnus, W., 1974. Non euclidean tesselations and their groups. Academic Press, New York.

[10] Ratclife, J. G., 1994. Foundations of hyperbolic manifolds., Vol. 149, Springer Verlag. New York.

[11] Stillwell, J., 1996. Sources of hyperbolic geometry. Board. Providence.

Received: July 12, 2006 